\title{
OTIONOMICS
}

Revista de los Estudios de Economía y Empresa

\section{Fvaluación de los estados financieros para determinar los factores clave que afectan a la misión de Adobinue, S. L.}

\author{
Maria Francisca Manrique Pérez \\ Financial Controller en Adobinve, S. L. \\ Alumni Grado en Administración y Dirección de Empresas \\ Estudios de Economía y Empresa (UOC)
}

RESUMEN Este artículo es una síntesis de mi Trabajo Final de Grado1 en Administración y Dirección de Empresas, concretamente en el ámbito de gestión contable y financiera, donde he realizado un análisis de una empresa del sector del curtido de la piel, con el objetivo de determinar los factores clave que afectan a su misión. Con esta finalidad, he realizado un análisis cuantitativo (económicofinanciero) y cualitativo de la organización, del sector donde opera y finalmente, de las variables macroeconómicas de su entorno.

La empresa objeto de mi TFG es Adobinve, S. L., dicha empresa se dedica a prestar los servicios de ribera para las pieles de ovino y caprino con el destino final de la confección, su ámbito de actuación es nacional y dentro de la comunidad europea.

He considerado más adecuado enfocar este artículo desde la metodología utilizada en el estudio integral de la empresa para localizar los factores clave para la consecución de su misión "ser la ribera de Europa", al tener el convencimiento de que puede ser de mejor ayuda para futuros TFG en el ámbito de gestión contable y financiera.

PALABRAS CLAVE misión; factores clave; ratios; indicadores; DAFO; sector

\section{Assessment of Financial Statements to Identify Key Factors in the Target of Adobinve, S.L.}

ABSTRACT This article is a synthesis of my final degree project ${ }^{1}$ in Administration and Management, specifically in the area of Accounting and Financial Management, where I have analyzed a company in the leather tanning industry with the aim of determining the key factors towards meeting its target. For this purpose, I have done a quantitative (economic and financial) and qualitative analysis of the company, the industry it operates in and the macroeconomic variables of its environment. 
The company studied in my FDP is Adobinve, S.L. It is a tanning company with a national and EU-wide scope, specializing in beamhouse operations with sheep and goat leather for the clothing industry.

For this article, I have deemed more appropriate to take the methodology used in the comprehensive study of the company in order to single out the key factors to meet its "Being Europe's skin beamhouse" target. I am sure this can be helpful for future FDPs within Accounting and Financial Management.

KEYWORDS target; key factors; rates; indicators; SWOT; industry

\section{Introducción}

El objetivo de mi TFG ha sido evaluar los factores clave que afectan a la misión de Adobinve (ser la ribera de Europa). Con esta finalidad, realicé un análisis integral de la empresa, desde la vertiente interna, donde la valoré tanto cuantitativa como cualitativamente y, desde la vertiente externa, donde analicé el sector en el que opera y su entorno económico, político y social. Con los datos obtenidos obtuve suficiente información para poder realizar un diagnóstico integral con sus recomendaciones y posterior simulación, finalizando mi trabajo con las conclusiones a las que llegué, así como las implicaciones éticas y sociales que podía tener la consecución de la misión de Adobinve.

A continuación enumero el alcance de mi TFG:

- Diagnóstico cuantitativo y cualitativo de Adobinve mediante el análisis de las cuentas anuales disponibles de cinco ejercicios auditados sin salvedades y otras fuentes.

- Estudio del sector, comparando variables clave obtenidas de las CC. AA. disponibles, de una serie de empresas seleccionadas mediante criterios preestablecidos, complementando la información obtenida con los datos de la industria textil y del curtido disponible en la central de balances del Banco de España.

- Estudio del sector ganadero del ovino debido a su incidencia sobre el sector de la piel.

- Valoración de la situación y perspectiva del entorno político, económico y social, con el análisis de los datos macroeconómicos que podían afectar a las empresas ubicadas a España.

- Descripción de la situación de Adobinve al cierre del último ejercicio analizado.

- Elaboración de una matriz DAFO (debilidades, amenazas, fortalezas y oportunidades).

- Elaboración de un diagrama causa-efecto relacionando los puntos fuertes y débiles con sus causas.

- Diagnóstico integral detectando los factores clave que afectan a la misión de la organización.

- Propuesta de medidas para solucionar los problemas detectados con la simulación de las posibles consecuencias sobre los factores clave de la misión y de los estados financieros de la empresa.

- Reflexión sobre el impacto social y ético de la misión de la empresa.

- Conclusión. 


\section{Análisis interno de la empre}

\subsection{Análisis cuantitativo}

Para realizar el análisis cuantitativo de la empresa, obtuve la memoria de las cuentas anuales de los cincos últimos ejercicios disponibles en el Registro Mercantil, que estaban auditadas sin salvedades, dotándolas de mayor confianza a la hora de ser analizadas.

Con la información obtenida elaboré una valoración de la situación económico-financiera de Adobinve, S. L., valorando la capacidad de la empresa a la hora de conseguir su misión, y utilizándola como punto de partida para la propuesta.

La metodología de trabajo que utilicé para el análisis interno fue:

- Análisis patrimonial mediante el método de porcentajes verticales y horizontales de las CC. AA.:

- Balance.

- Cuenta de pérdidas y ganancias.

- Estados de cambio en el patrimonio neto.

- Estado de flujos de efectivo.

- Cálculo del fondo de maniobra:

- Fondo de maniobra aparente (activo corriente - pasivo corriente).

- Fondo de maniobra necesario (activo corriente de explotación - pasivo corriente de explotación).

- Comparativa entre FM aparente y FM necesario, y valoración de la solvencia a corto plazo de la empresa.

- Análisis financiero y económico a través de ratios:

- Endeudamiento.

- Solvencia a corto plazo.

- Gestión de cobro y pagos.

- EVA (valor económico añadido) y WACC (coste ponderado del capital).

- Rentabilidad económica.

- Productividad.

- Predicciones mediante el enfoque unidimensional y multidimensional:

- Enfoque unidimensional: para complementar la información obtenida, con los métodos expresados en este apartado, complementé los datos utilizando las 5 ratios con más poder predictivo, según el estudio realizado sobre más de 80.000 empresas españolas y publicado por el Dr. Oriol Amat. En la tabla $1.1 \mathrm{se}$ muestran estas ratios.

\section{Tabla 1.1. Ratios con más poder predictivo}

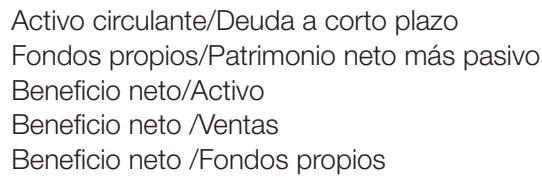

Fuente: Amat (2008)

- Enfoque multidimensional: Son varios los estudios que utilizan un indicador sintético para diferenciar las empresas saneadas de las que están en peligro, los más conocidos son la fórmula Z de Edward Altman o el índice de Higgins. Para mi trabajo consideré más idóneo utilizar la adaptación, realizada por la UPF, de la fórmula Z de Altman. 


\subsection{Análisis cualitativo}

Para realizar un análisis interno completo, es importante tener en cuenta también su intangible a través de una valoración cualitativa, en este caso tuve en cuenta tres factores:

- Los recursos humanos, ¿quién?

- Aspectos estratégicos, ¿qué hace la empresa?

- Aspectos operativos, ¿cómo lo hace la empresa?

\section{Análisis externo}

Adobinve actúa en un sector muy maduro. España tiene una gran tradición milenaria en el arte del curtido, lo que la convierte en la segunda productora de piel curtida de Europa. Su gran consumo de mano de obra y agua, con su correspondiente impacto medioambiental, ha provocado que parte de la producción se haya trasladado a países con costes bajos de mano de obra y con una legislación más flexible en medio ambiente. Las empresas que han continuado en Europa han tenido que invertir en tecnología para mejorar los procesos productivos y minorar su impacto medioambiental. Adobinve posee unas magníficas instalaciones para el cuidado de nuestro entorno, dotándola de un gran valor añadido delante de la competencia.

\subsection{Análisis del sector del curtido de ovino para la confección}

Antes de proceder al estudio del sector del curtido del ovino para la confección, analicé la evolución y la calidad de la piel ovina española por su vital importancia en la continuidad del sector del curtido.

Evolución de la calidad y cantidad de la piel ovina española

Las pieles españolas han perdido calidad en los últimos años a causa de:

- Malas prácticas relacionadas con la cría y engorde de los animales.

- Malas prácticas en los mataderos.

- Defectos relacionados con una no correcta conservación y manipulación de las pieles en los almacenes.

- La menor cantidad de pieles disponibles con la disminución de la cabaña y sacrificio de ovejas. En el gráfico 1 se muestra la descendencia del sacrificio de ovino en España, donde se muestra un descenso muy significativo de las pieles disponibles para el sector de la curtición.

Gráfico 1. Sacrificio de ovino en España

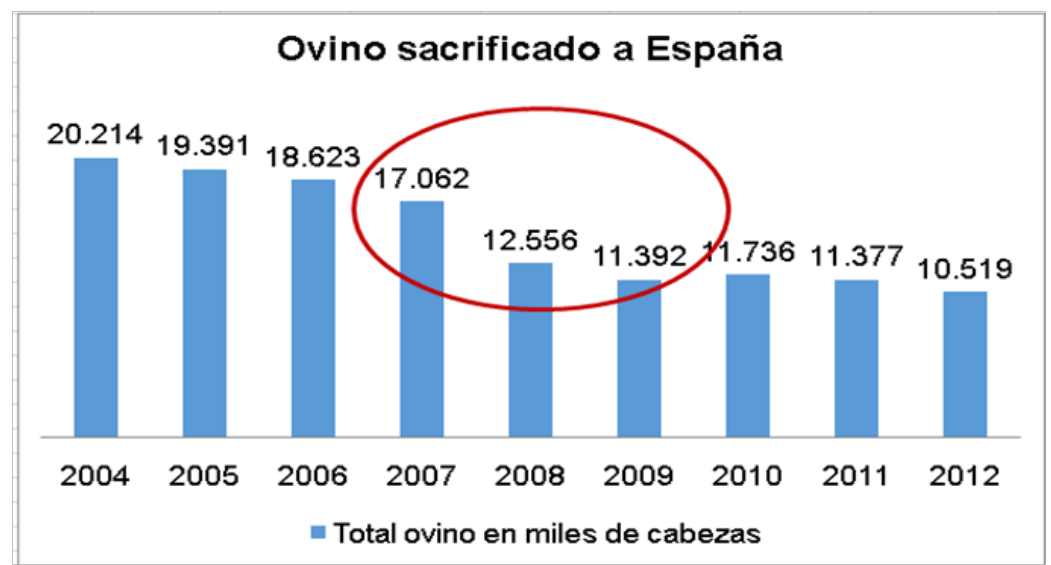

Fuente: Elaboración propia, datos del Ministerio de Agricultura, Alimentación y Medio Ambiente. 


\section{Criterio de selección de la muestra del sector}

A la hora de analizar el sector donde opera una empresa, es muy importante hacer una correcta selección de las empresas a analizar. En un sector con muchos agentes es aconsejable realizar una muestra que represente al sector.

Los criterios de selección que consideré más oportunos para mi estudio fueron los tipos de piel, ovino, y su destino final, la confección. La información la obtuve de la Web de ACEXPIEL (www.leather-spain.com). Esta asociación tiene adscritas 111 empresas que representan el 90\% de la producción nacional. En su web están disponibles las tablas de las empresas adscritas por tipos de piel y destino final, donde obtuve un total de 14 empresas para comparar su evolución con la de Adobinve.

Para poder comparar las empresas seleccionadas con Adobinve, tuve que acceder a las cuentas anuales de los cincos ejercicios analizados en mi trabajo a través del SABI (Sistema de Análisis de Balances Ibérico).

\section{Posición de Adobinve en el sector}

Una vez trabajados los datos de las 14 empresas analizadas, escogí una serie de indicadores que fuesen comparables entre todas las empresas, como son la cuota de mercado, la fórmula Z, el BAllAD (beneficio antes de intereses, impuestos, amortizaciones y dotaciones), el BI (beneficio antes de impuestos), así como los gastos financieros.

Con estos datos llegué a la conclusión de que Adobinve es muy sensible a la tendencia del sector, aunque tiene un comportamiento mejor gracias a una correcta implantación de un sistema de control de gestión.

Para complementar la información del sector, amplié los datos obtenidos con los datos disponibles en la Central de Balance del Banco de España sobre la industria textil de la confección y del cuero, escogiendo indicadores que se puedan comparar entre empresas del mismo sector pero con diferentes características, estos indicadores fueron su rentabilidad económica y financiera, el coste financiero y su endeudamiento.

\subsection{Análisis del entorno político, económico y social}

Para poder conocer mejor el entorno donde opera cualquier empresa, se ha de complementar el análisis del sector con los datos macroeconómicos que nos informa del entorno político, económico y social. En mi trabajo analicé los siguientes aspectos:

- Estabilidad política y actitud gubernamental hacia las empresas:

El periodo analizado en este estudio (2008-12) se caracteriza por una crisis económica, social y política, donde consumidores y empresas han perdido la confianza en el gobierno.

- Crecimiento del país, los indicadores que nos informan del crecimiento, estancamiento o decrecimiento de un país son:

- El PIB (producto interior bruto).

- La inflación.

- La evolución de los tipos de interés.

- El IPC (índice de precios del consumo).

- Efecto social de la crisis, los indicadores que nos informan de los efectos sociales durante el periodo estudiado son:

- La tasa de paro.

- El déficit de las administraciones públicas.

Los datos macroeconómicos los obtuve del Instituto Nacional de Estadística y del Eurostat.

Para finalizar, complementé el estudio del entorno con la evolución del mercado de lujo, al ser este el destino final de las pieles curtidas en la empresa. 


\section{Diagnóstico}

\subsection{Análisis DAFO y diagrama causa-efecto}

Con los datos obtenidos mediante el análisis interno y externo obtuve suficiente información para poder realizar una matriz DAFO, representando las características internas mediante las debilidades y fortalezas, y las externas mediante las amenazas y oportunidades.

La matriz DAFO me sirvió de base para la confección del diagrama causa-efecto, donde relacioné las debilidades, amenazas, fortalezas y oportunidades entre sí y su efecto sobre la empresa.

\subsection{Diagnóstico y factores clave}

Llegados a este punto, se puede realizar un diagnóstico de la empresa a su cierre 31/12/2012 y determinar cuáles son los factores clave para la consecución de su misión "ser la ribera de Europa".

- Diagnóstico a 31/12/2012:

- Impacto medioambiental.

- EDAR y vertedero propio.

- Agua, pozos propios.

- Altos consumos.

- Pérdida de calidad y cantidad del ovino español.

- ABC clientes peligroso.

- Poco endeudada.

- Buena liquidez.

- Especializada solo en un tipo de piel.

- Factores claves:

- MEDIO AMBIENTE: agua, EDAR (estación depuradora de aguas residuales), vertedero, fiscalidad medioambiental.

- Calidad del ovino español.

- Diversificación en otros tipos de pieles.

- I+D+i para reducir los consumos: innovación en inmovilizado, mejora en las fórmulas, mejora de los procesos.

- Ampliar el ABC de clientes.

- Pasar costes fijos a variables.

\section{Recomendaciones}

Basándome en mi estudio y en que la misión de Adobinve es encabezar la investigación y la innovación con el objetivo de convertirse en un modelo de gestión SOSTENIBLE, EFICIENTE Y SEGURO, llegué a la conclusión de que el factor clave más importante era ser una empresa VERD. Por este motivo propuse invertir en I+D+i aprovechando la buena liquidez y apalancamiento obtenidos del análisis de las cuentas anuales.

Concretamente, mi propuesta consistía en una inversión con el objetivo de conseguir mejorar el consumo de mano de obra directa, de aprovisionamiento (productos químicos) y otros gastos de explotación con gran impacto medioambiental (agua, luz, gas y tributos medio ambientales), haciendo una simulación de esta propuesta a cinco años vista. 


\section{Simulación de las recomendaciones}

A la hora de realizar la simulación, partí de las últimas cuentas anuales analizadas y tuve en cuenta la información obtenida en la memoria y en las ratios efectuadas sobre los estados financieros para poder hacer una proyección a cinco años vista.

La simulación constaba de:

- Balance provisional.

- Cuenta de pérdidas y ganancias provisional.

- Estado de flujos de efectivo provisional.

- Ratios sobre las cuentas anuales provisionales.

\section{Implicaciones éticas y/o sociales}

La creciente preocupación de las sociedades desarrolladas por el entorno ha puesto en marcha, en las empresas punteras y en la sociedad, lo que se conoce por una contabilidad medioambiental en la fabricación de productos, la EP\&LA (Environmental Profit and Loss Account).

Temas como la emisión de gases de efecto invernadero, la reducción de la emisión de carbono, la reducción en el consumo de agua y consumos energéticos son los nuevos conceptos que persiguen un snowball effecte en la cadena de proveedores de un determinado artículo. Adobinve no es indiferente a este movimiento, por lo que trabaja para adaptar sus procesos a las exigencias de una nueva contabilidad verde, su capacidad de adaptación o no, será el factor clave más decisivo para continuar produciendo en un futuro, es por eso, por lo que en la propuesta consideré invertir en maquinaria e instalaciones para mejorar la relación de la empresa con el medio ambiente.

\section{Bibliografia}

ACEXPIEL (www.leather-spain.com)

ADOBINVE, S.L. (www.adobinve.com)

ALEMANY, N.; VILADECANS, C. (2011). Análisis de los estados de cambio en el patrimonio neto y de los estados de flujo de efectivo. Barcelona: ACCID.

AMAT, O. (2008). Análisis Económico-Financiero. Barcelona: Gestión 2000.

AMAT, O. (2008). Análisis integral de empresas. Barcelona: Bresca Editorial, S.L.

BANCO CENTRAL DE ESPAÑA, central de balances (http://www.bde.es/bde/es/areas/cenbal/),

BRUN, X.; LARRAGA, P.; MOYA, M. (2010). Cómo interpretar la información económica. Barcelona: Profit Editorial I., S. L.

CAMPA, F. (2009). Análisis de la rentabilidad de la empresa. FUOC: PID_00142063.

CONFEDERACIÓ ESPANYOLA D'ADOBADORS CEC-FECUR. (2005). La indústria de l'adob, de l'art a la tecnologia. Barcelona: Curtidores Españoles.

EUROSTAT (http://epp.eurostat.ec.europa.eu/portal/page/portal/eurostat/home)

INSTITUTO NACIONAL DE ESTADÍSTICA (http://www.ine.es).

LEY 15/2010, de 5 de julio, de modificación de la Ley 3/2004 de Morosidad.

LOEWE, calidad de la piel española (http://www.loewe.com/eu_es/tradicion-y-origines/la-piel-de-loewe.html), data de acceso 21/04/2014.

MINISTERIODEAGRICULTURA, ALIMENTACIÓNYMEDIOAMBIENTE, datosestadísticos, encuestaganadera2013 (http://www.magrama.gob.es/es/estadistica/temas/estadisticas-agrarias/ganaderia/encuestas- 
ganaderas/\#para4) y encuesta sacrificio 2013 (http://www.magrama.gob.es/es/estadistica/temas/estadisticas-agrarias/ganaderia/encuestas-sacrificio-ganado/) fecha de acceso 21/04/2014.

PLAN GENERAL DE CONTABILIDAD, Real Decreto 1514/2007.

VALOR AFEGIT, (http://www.tv3.cat/videos/5055971/Es-traspassa-experiencia), fecha de acceso 25/04/2014.

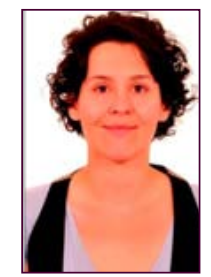

Maria Francisca Manrique Pérez

mmanriquep@uoc.edu

Alumni Grau ADE

Graduada en Administración y Dirección de Empresas por la Universitat Oberta de Catalunya (UOC), máster universitario de Dirección Financiera y Contable, especialidad de auditoría en la Barcelona School of Management de la Universidad Pompeu Fabra, diplomada en Ciencias Empresariales por la Universitat Oberta de Catalunya y miembro de la comisión de control de gestión de l'Associació Catalana de Comptabilitat i Direcció (ACCID) adscrita al Colegio de Economistas. Actualmente desarrolla su carrera profesional como financial controller en Adobinve, S. L.

Los textos publicados en esta revista están -si no se indica lo contrario- bajo una licencia Reconocimiento-Sin obras derivadas 3.0 España de Creative Commons. Puede copiarlos, distribuirlos y comunicarlos públicamente siempre que cite su autor y la revista y la institución que los publica (autoría, nombre de la revista, institución editora); no haga con ellos obras derivadas. La licencia completa se puede consultar en http://creativecommons.org/licenses/by-nd/3.0/es/deed.es.

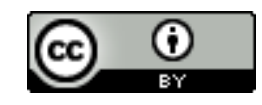

